\title{
Recidiva de Cisto Dermoide Congênito de Localização Paramediana
}

\section{Recurrence of Congenital Dermoid Cyst of Paramedian Location}

\author{
Estevam Rubens Utumi*, Camila Eduarda Zambon**, Irineu Gregnanin Pedron***, \\ Gustavo Grothe Machado****, André Caroli Rocha*****.

\begin{abstract}
* Mestre em Ciências Odontológicas com área de concentração em Clínica Integrada. Cirurgião Bucomaxilofacial da Força Aérea Brasileira, Hospital de Aeronáutica de São Paulo.

** Mestre em Ciências Médicas no Hospital das Clínicas da FMUSP. Especialista em Cirurgia Bucomaxilofacial.

**** Mestre em Ciências Odontológicas com área de concentração em Clínica Integrada. Periodontista do corpo clínico da Força Aérea Brasileira, Hospital de Aeronáutica de São Paulo.

***** Mestre em Ciências Médicas pelo Hospital das Clinicas da FMUSP. Chefe do Serviço de Cirurgia Traumatologia Bucomaxilofacial do Hospital das Clínicas da Faculdade de Medicina da USP.

****** Doutor em Diagnóstico Bucal pela Faculdade de Odontologia da Universidade de São Paulo. Assistente do Serviço de Cirurgia Traumatologia Bucomaxilofacial do Hospital das Clínicas da FMUSP.

Instituição: Hospital das Clínicas da Faculdade de Medicina da Universidade de São Paulo. São Paulo / SP - Brasil.

Endereço para correspondência: Estevam Rubens Utumi - Rua Pelotas, 284 - Apto. 21 - Vila Mariana - São Paulo / SP - Brasil - CEP: 04012-000 - Telefone: (+55 11) 5549-8241 - E-mail: estevamutumi@uol.com.br

Artigo recebido em 29 de Abril de 2009. Artigo aprovado em 24 de Maio de 2009.
\end{abstract}

\section{RESUMO}

Introdução:

Palavras-chave:

O cisto dermoide é um cisto de desenvolvimento incomum na face e envolve mais frequentemente o assoalho bucal. A maioria das lesões ocorre em adultos jovens, com ligeira predileção pelo gênero masculino. As lesões neonatais e em crianças são extremamente raras. Manifesta-se como tumefação flutuante, assintomática e de crescimento lento e progressivo, atingindo dimensões variadas. Sua cápsula pode conter um ou mais anexos cutâneos, como glândulas sebáceas, pêlos ou unhas. O tratamento é cirúrgico, através de enucleação, sendo sua recidiva incomum. O aspecto clínico, histopatológico, diagnóstico diferencial e tratamento de um caso de cisto dermoide congênito, e de sua recidiva, são discutidos pelos autores. Palavra chave: cisto dermoide; soalho de boca; recorrência. cisto dermoide, soalho bucal, recidiva.

\section{SUMMARY}

Introduction:

The dermoid cyst is a cyst of unusual development in the face and more often involves the mouth floor. Most injuries occur in young adults with a slight predilection for males. Lesions in neonatal and children are extremely rare. It manifests as swelling floating asymptomatic and slow-growing and progressive, reaching dimensions. His capsule may contain one or more skin appendages such as sebaceous glands, hair or nails. The treatment is surgical, by enucleation, and its recurrence uncommon. The clinical appearance, histopathology, differential diagnosis and treatment of a case of congenital dermoid cyst, and its recurrence, are discussed by the authors.

Keywords: dermoid cyst, mouth floor, recurrence. 


\section{INTRODUÇÃO}

O cisto dermoide é uma alteração de desenvolvimento relativamente rara na região de cabeça e pescoço. Quando na boca, sua localização mais frequente é o assoalho bucal. Há ligeira predileção pelo gênero masculino e a maioria das lesões ocorre entre a segunda e terceira décadas de vida. As lesões congênitas e as originárias em crianças são extremamente raras (1-7).

Apresenta-se comumente como uma tumefação flutuante na porção anterior do assoalho de boca, com crescimento lento, progressivo e indolor, podendo variar o tamanaho $(3,4)$.

O tratamento dos cistos dermoides de soalho bucal é cirúrgico, através da enucleação. Até o momento, há poucos casos de transformação maligna (8) e nenhuma recidiva relatada. Os autores relatam um caso de cisto dermoide com localização sublingual paramediana, e sua recidiva após enucleação em um recém-nascido.

\section{Caso Clínico}

Paciente F.C.S. de 10 dias de vida, leucoderma, gênero feminino foi encaminhada pelo médico pediatra ao nosso serviço para avaliação de tumefação em assoalho bucal que foi percebida logo após o nascimento. Havia dificuldades na sucção durante a amamentação e deglutição.

Ao exame físico, apresentava-se em bom estado geral e sem alterações relevantes na história médica pregressa e antecedentes hereditários. Ao exame intraoral, observou-se crescimento nodular submucoso em região paramediana à esquerda, flutuante à palpação, medindo cerca de $1 \mathrm{~cm}$ no seu maior diâmetro. A mucosa recobrinte possuía coloração e textura normais (Figura 1). Com hipótese diagnóstica foi de rânula, optou-se pelo acompanhamento. Após 3 meses, não houve involução, ocorrendo um aumento progressivo do nódulo, com manutenção das características descritas anteriormente. Neste momento, solicitou-se uma tomografia computadorizada com contraste, que mostrou imagem cística, cruzando a linha média, situada entre os músculos genio-hioideos e deslocada para esquerda no seu aspecto mais superior. A cápsula era espessada e o conteúdo hipoatenuante em relação aos tecidos moles adjacentes do assoalho bucal (Figuras 2). O diagnóstico presuntivo foi de cisto dermoide.

Foi realizada a biópsia excisional, sob anestesia geral e intubação nasotraqueal. Iniciou-se com uma incisão intraoral longitudinal em soalho bucal, sobre a lesão, seguindose com divulsão romba dos tecidos adjacentes (Figura 3A).

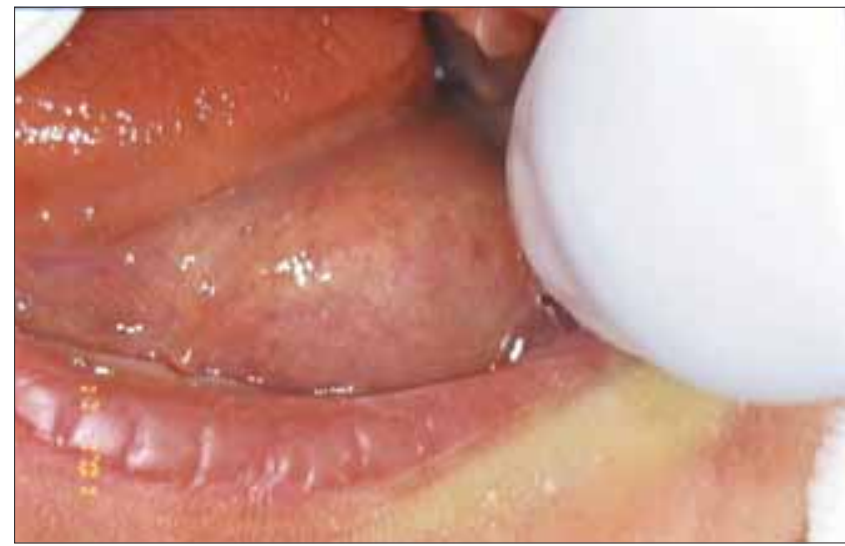

Figura 1. Mucosa da cavidade oral e visualização da lesão.

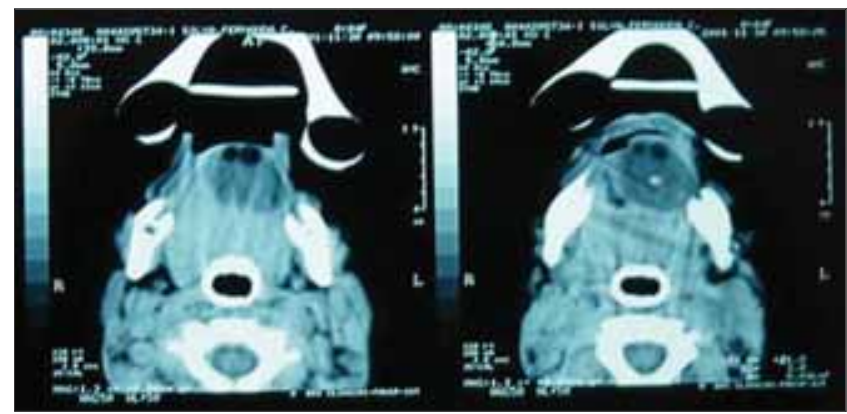

Figura 2. Tomografia computadorizada.

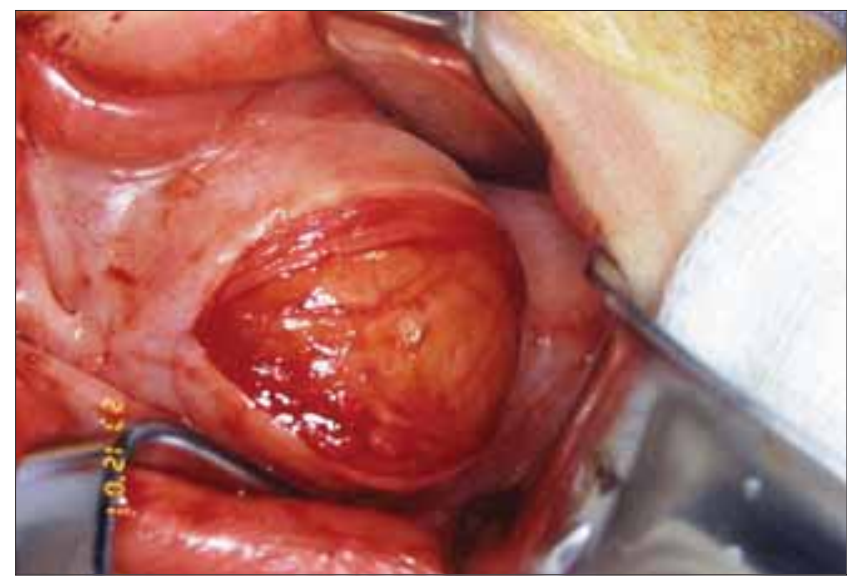

Figura 3a. Exerece da lesão.

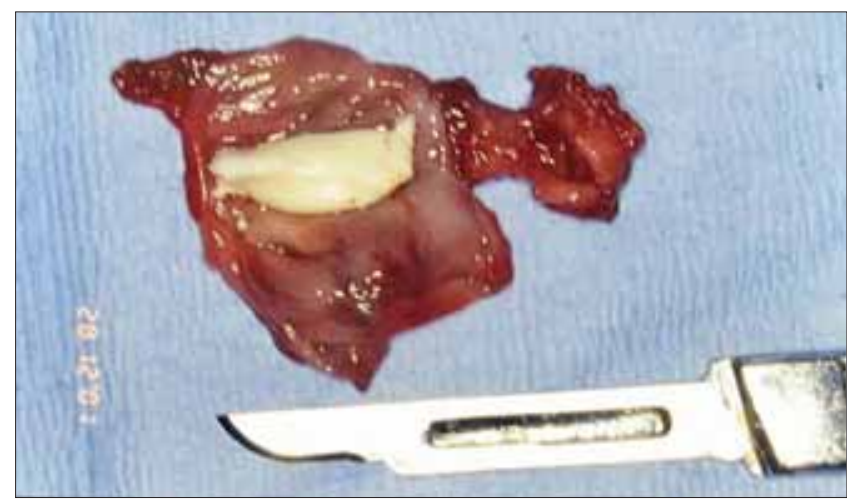

Figura 3b. Lesão bucal. 


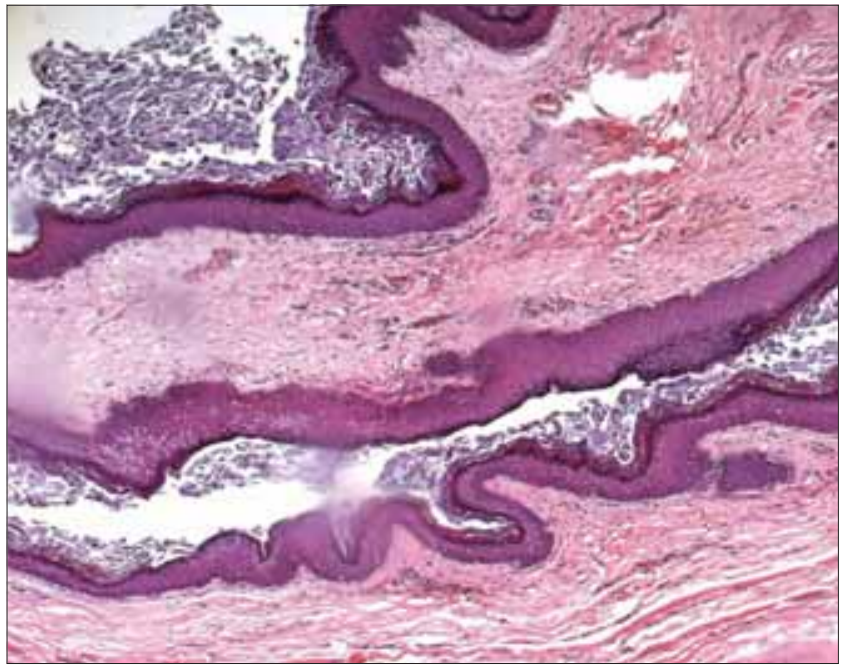

Figura 4a. Corte histopatológico.

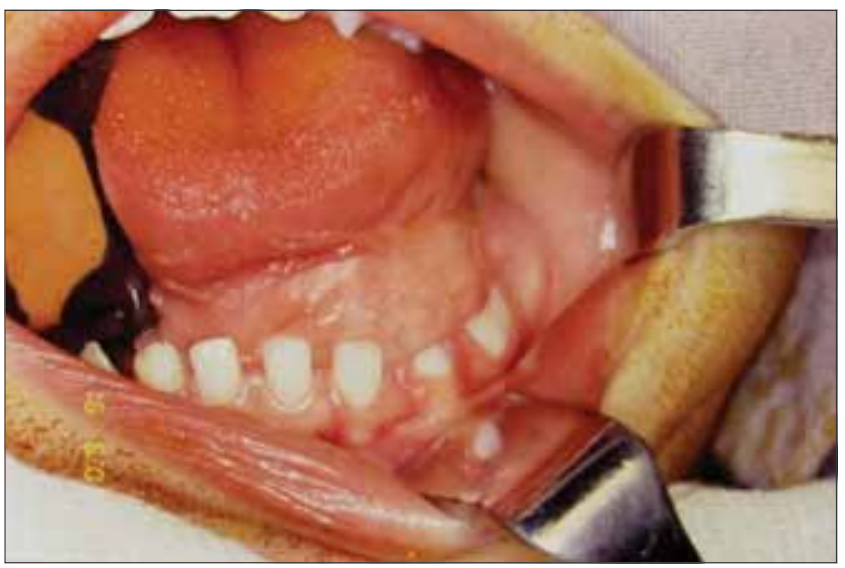

Figura 5. Aspecto pós-operatório

Ao final da dissecção, houve ruptura da cápsula cística e extravasamento de conteúdo pastoso de coloração esbranquiçada (Figura 3B). A remoção foi finalizada, não se visualizando lesão residual macroscopicamente na loja cirúrgica.

O exame histopatológico mostrou cápsula cística revestida por epitélio escamoso estratificado com queratinização, além de glândulas cutâneas anexas. Ligeiro infiltrado inflamatório mononuclear era visto em área esparsa (Figura 4A e B).

O pós-operatório transcorreu bem, porém com 1 ano de acompanhamento constatou-se novo aumento volumétrico na mesma região (Figura 5). Outra tomografia computadorizada foi realizada, demonstrando duas áreas císticas bem delimitadas em assoalho bucal, de limites espessados e com conteúdo hipoantenuante em relação aos tecidos moles circunvizinhos. (Figura 6). Uma nova cirurgia, sob anestesia geral, foi realizada para enucleação

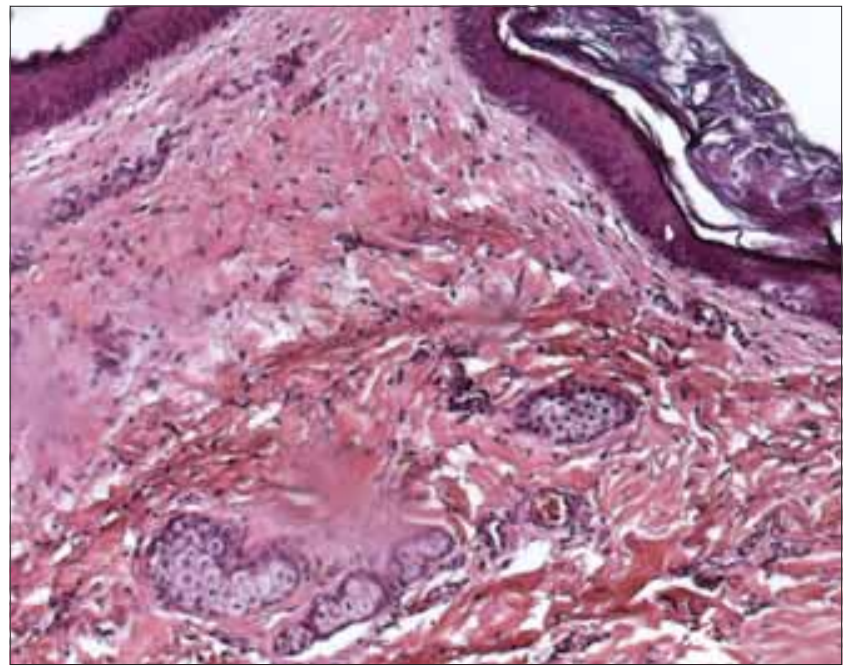

Figura 4b. Corte histopatológico.

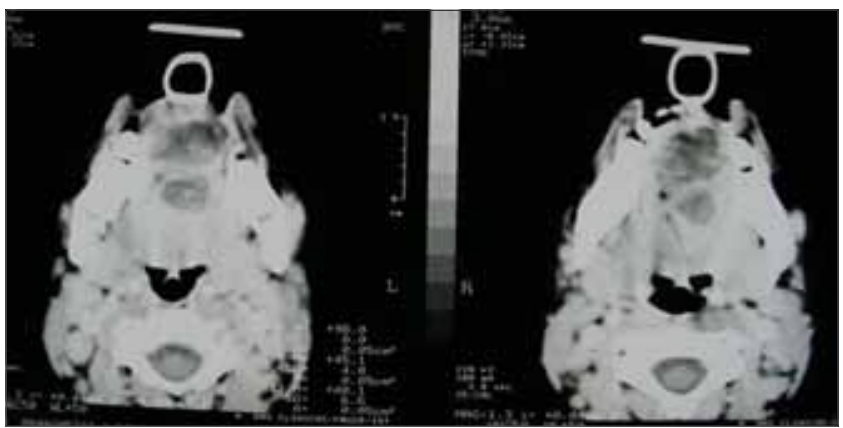

Figura 6. Tomografia computadorizada de controle.

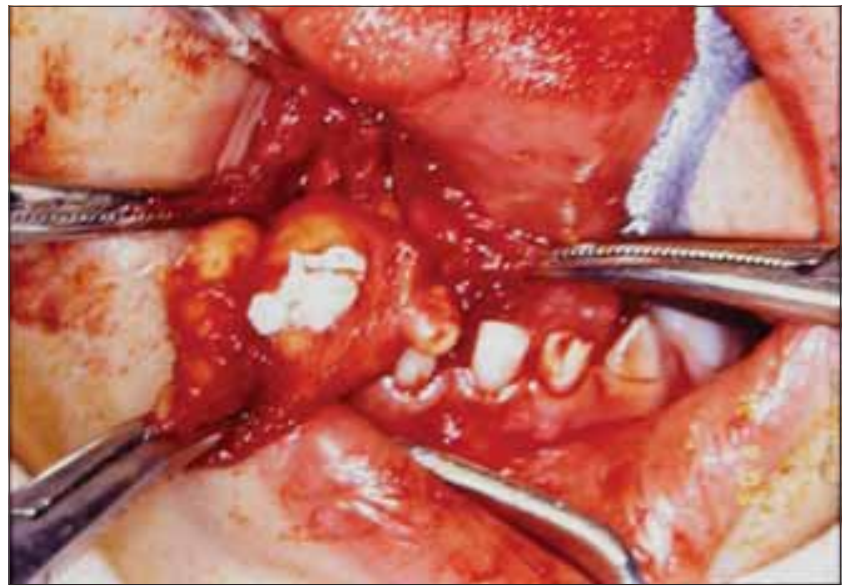

Figura 7. Exerese de uma segunda lesão.

do cisto (Figura 7). A incisão realizada foi vertical, em linha média de ventre lingual e assoalho bucal. A lesão foi removida e mostrava conteúdo pastoso, denso e esbranquiçado. O diagnóstico histopatológico foi novamente de cisto dermoide, semelhante ao resultado anterior. A paciente encontra-se bem e sem recidiva após 4 anos de seguimento da última intervenção. 
O primeiro caso de cisto dermoide sublingual foi descrito na Jordânia, em $1778(5,7)$. Localizações comuns incluem a órbita, dorso do nariz e pescoço (1). Estas lesões são raras na boca, mas quando ocorrem, geralmente são encontradas na porção mediana e anterior do assoalho bucal $(2,7,8,9)$. A localização paramediana vista neste caso corroborou para um diagnóstico tendencioso de rânula que só foi corrigido com a observação do padrão evolutivo da lesão que não suportou a hipótese diagnóstica inicial.

Poucos casos foram relatados em crianças ou idosos, além de extremamente raros em neonatais $(1,3,4,10)$. Não há predileção por sexo, apesar de um estudo mostrar mais homens afetados (6). A etiologia mais provável do cisto dermoide de soalho bucal é o aprisionamento de tecido ectodérmico na linha média, no momento da fusão do primeiro (mandibular) e segundo (hioide) arcos branquiais $(3,11)$. No presente caso, a lesão era congênita, dificultando o diagnóstico inicial.

A classificação dos cistos dermoides é baseada tanto na localização anatômica como no aspecto histopatológico. Meyer, em 1955, separou os cistos dermoides em três tipos: (1) cisto epidermoide - com parede limitada por epitélio escamoso estratificado sem estruturas anexas; (2) cisto dermoide - limitada por epitélio escamoso estratificado com anexos dérmicos, como glândulas sebáceas e sudoríparas e folículos pilosos no tecido conectivo subjacente e (3) teratoma - cavidade limitada por epitélio com derivados do mesoderma e endoderma, como músculo, mucosa intestinal, mucosa respiratória, fibras, osso, vasos sanguíneos, além dos anexos dérmicos típicos do cisto dermoide $(3,6,13,14)$. Já em 1969, Katz e Passy classificaram anatomicamente os cistos dermoides e epidermoides do assoalho bucal como: (1) sublingual - localizado na linha média, sob a língua, entre os músculos geniohióideo e milohióideo, sendo que, quando atinge grandes proporções, a língua é deslocada para a orofaringe; (2) geniohióideo localizado na linha média na região submentual entre a pele e os músculos geniohióideo, levando a uma aparência de queixo duplo e (3) cisto lateral - localizado na região submandibular e, normalmente, aumenta de tamanho em direção inferior ao osso hioide ou comprime superiormente o assoalho bucal, forçando a língua para o lado oposto (12). O tipo histológico da lesão parece não influenciar o prognóstico do caso, contudo a localização, se acima ou abaixo do músculo geniohióideo, pode dificultar a exérese pela via intra-oral, além de aumentar o risco de recidivas caso uma remoção incompleta seja realizada (3). Por isso, BLoom et al. (2002) (1) recomendaram acessos duplos, intra e extra-oral, em casos de cistos dermoides laterais. Além disso, em recém-nascidos, a localização sublingual pode prejudicar a alimentação e a respiração, produzindo alterações sistêmicas secundárias à patologia inicial $(5,11)$. Desta forma, uma classificação anatômica deve ser utilizada para auxiliar o planejamento cirúrgico e o prognóstico (4). Neste caso, mesmo com a impressão cirúrgica de remoção completa da lesão inicial, a ruptura da cápsula parece ter predisposto a permanência de lesão residual em algum nível. Com 1 ano de evolução, este remanescente evoluiu para a formação de nova área cística evidente clinicamente. De qualquer forma, a ocorrência congênita, o novo crescimento e o padrão tomográfico, sugere tratar-se de uma lesão de comportamento mais agressivo.

Como diagnóstico diferencial deve-se considerar, infecção ou celulite submandibular e sublingual, rânula, bloqueio uni ou bilateral do ducto de Wharton, higroma cístico, cisto branquial, cisto do ducto tireoglosso, massa gordurosa em área submental, neoplasia sublingual ou de glândula salivar menor, lipoma, fibroma, hemangioma ou linfangioma e tumor maligno $(6,7,9,15,16)$. Os exames complementares incluem ultrassonografia, tomografia computadorizada e ressonância nuclear magnética. Estes permitem a visualização e localização precisa da lesão em relação às estruturas anatômicas adjacentes, além de auxiliar na escolha da técnica cirúrgica mais apropriada (3). Este recurso proporcionou a visualização da parte mais profunda da lesão, que se localizava na linha média, entre os músculos geni-hióideos, contribuindo para o diagnóstico de cisto dermoide.

O acesso intra-oral é utilizado para a maioria dos casos. Entretanto, lesões localizadas abaixo do músculo milo-hióideo devem ser abordadas por acesso extra-oral $(4,13)$. Apesar de tratar-se de uma lesão de ótimo prognóstico, o acompanhamento pós-operatório de, no mínimo três anos deve ser realizado em todos os casos. A evolução deste caso reforça esta necessidade.

\section{COMENTÁRIOS FINAIS}

O cisto dermoide é uma lesão de ocorrência incomum na boca. Sua manifestação congênita e localização atípica (paramediana) levaram a maior dificuldade diagnóstica e terapêutica. Detalhes tomográficos e evolução clínica definiram o momento mais adequado para abordagem cirúrgica. O acompanhamento pós-operatório deve ser realizado sempre.

\section{REFERÊNCIAS BIBLIOGRÁFICAS}

1. Bloom D, Carvalho D, Edmonds J. Neonatal dermoid cyst of the floor of the mouth extending to the midline neck. Arch Otolaryngol Head Neck Surg. 2002, 128:68-7.

2. Bodner L, Woldenberg Y, Sion-Vardy N. Dermoid cyst of the maxilla. Int J Oral Maxillofac Surg. 2004, 34: 453-55. 
3. Eplley BL, Bell MJ, Sclaroff. Simultaneous occurrence of dermoid and heterotopic intestinal cysts in the floor of the mouth of a newborn. J Oral Maxillodac Surg. 1985, 43(11): 880-3.

4. Gibson WS, Fenton NA. Congenital sublingual dermoid cyst. Arch Otolaryngol. 1982, 108(11):745- 748.

5. Howell CJT. The sublingual dermoid cyst. Report of five cases and review of the literature. Oral Surg Oral Med Oral Pathol. 1980, 59:578-580.

6. Longo F et al. Midline (dermoid) cysts of the floor of the mouth: report of 16 cases and review of surgical techniques. Plast Reconst Surg. 2003, 112(6):1560-65.

7. Oygur et al. Oral Congenital dermoid cyst in the floor of the mouth of newborn. Oral Surg. 1992, 74(4-6):627-630.

8. Devine JC, Jones DC. Carcinomatous transformation of a sublingual dermoid cyt. A case report. Int J Oral Maxillofac Surg. 2000, 29:126-7.

9. Howell CJT. The sublingual dermoid cyst. Report of five cases and review of the literature. Oral Surg Oral Med Oral Pathol. 1980, 59:578-580.
10. Nagar H, Baratz M. Congenital sublingual teratoid cyst. Case report. Int J Oral Maxillofac Surg. 1993, 22:44-45.

11. Ferran F, Abifadel M. Kyste dermoid du plancher buccal. Ann Chir Plast Esthét. 1990, 35(1):69-72.

12. Lowry RE, Tempero RM, Davis LF. Epidermoid cyst of the floor of the mouth. J Oral Surg. 1979, 37:271-73.

13. Ceccheti MM. et al. Intraoral enucleation of dermoid cyst- a case report. Rev Pós Grad. 2003, 10(1):88-93.

14. Seah T, Sufyan W. Case report of a dermoid cyst at the floor of the mouth. Ann Acad Med Singapore. 2004, 33(4):7779.

15. Janjua TA, Goranvalingappa R. Quiz case 1 Submandibular dermoid cyst Arch Otolaryngol Head Neck 1990, 25(11):1270-1272.

16. Verrina G, Carta M. Considerazioni sulle cisti dermoidi del pavimento della bocca. Minerva Stomatol. 1989, 38(16):683-686. 